\title{
The Impact of Anterior Urethropexy During Robotic Prostatectomy on Urinary and Sexual Outcomes
}

\author{
Emilie K. Johnson, M.D., Ryan C. Hedgepeth, M.D., Chang He, M.S., and David P. Wood, Jr., M.D.
}

\begin{abstract}
Objectives: We determined the effect of an anterior urethropexy (AU) stitch on postoperative urinary continence, irritative urinary symptoms, and sexual function after robotic radical prostatectomy (RP).

Methods: Consecutive patients undergoing robotic RP for prostate cancer were prospectively evaluated. The Expanded Prostate Cancer Index Composite (EPIC) questionnaire was administered pre- and postoperatively to all patients. Patients were then grouped by AU status. A linear mixed model was used to compare the rate of recovery in incontinence (UIN), irritative (UIR), and sexual domain scores between the two groups. A $t$-test was used to compare UIN, UIR, and sexual domain scores at specific time points.

Results: A total of 229 patients underwent robotic RP and filled out a preoperative and at least one postoperative EPIC questionnaire. In this population, 87 did have and 142 did not have an AU performed. The mean EPIC-UIN score at 3 months was 68 in the AU group and 58 in the non-AU group $(p=0.015)$. Comparison of all other time points and overall urinary scores revealed no other statistically significant differences after surgery. Sexual domain scores were also improved at 3 months in the AU group $(p=0.002)$.

Conclusions: AU during robotic RP leads to improved urinary continence and sexual functioning at 3 months of follow-up. An earlier return to continence may facilitate an earlier return to sexual activity. AU may offer a shortterm quality-of-life advantage for patients undergoing robotic RP.
\end{abstract}

\section{Introduction}

$\mathbf{T}$ HE PRIMARY GOAL of radical prostatectomy (RP) for localized prostate cancer is oncologic control. Most patients who undergo the procedure for clinically localized prostate cancer can expect to be disease-free for the rest of their life. Although prostatectomy offers an excellent chance of disease cure, it also impacts urinary continence and sexual function. ${ }^{1}$ Incontinence and erectile dysfunction have important costs, both financially and in terms of their impact on health-related quality of life (HRQOL). Patients with incontinence use disposable pads regularly and may ultimately undergo treatment with an artificial urinary sphincter. ${ }^{2}$ HRQOL costs from incontinence may include changes in physical and social activity that cause patients stress. ${ }^{3}$ Patients with erectile dysfunction purchase erectile aids, medications, and live with the psychological stigma of impotence. Patient satisfaction with their chosen treatment for prostate cancer is significantly impacted by HRQOL outcomes along with cancer control. ${ }^{4,5}$

Various surgical techniques have been described to help improve HRQOL outcomes. Most notably, nerve sparing modifications have been developed to improve erectile function, and bladder neck sparing methods have sought to optimize continence. ${ }^{6,7}$ Several validated instruments for measuring HRQOL in men treated for prostate cancer are now available. These tools provide standardized ways to assess the impact that modifications in surgical technique have on patients' function and bother after treatment.

Understanding how surgical modifications affect urinary outcomes after prostatectomy can be a means for improving patient HRQOL, as well as minimizing the financial burden of incontinence and erectile dysfunction. The anterior urethropexy (AU) stitch has been advocated as one means of improving urinary outcomes after prostatectomy. The anatomic argument for $\mathrm{AU}$ is that it increases urethral support and re-creates the function of the cut puboprostatic ligaments. AU has shown promise in several series of open $\mathrm{RP},{ }^{8,9}$ as well as in one recent series of robotic RP. ${ }^{10}$ However, these studies did not evaluate the entire EPIC urinary domains or the effect of the AU stitch on sexual recovery. The purpose of this study is to examine the effect of an AU stitch on complete urinary and sexual outcomes after robotic RP.

\section{Methods}

Patient identification

A prospective evaluation of 229 consecutive patients undergoing robotic RP for prostate cancer from January 2006 to

Department of Urology, University of Michigan, Ann Arbor, Michigan. 
October 2008 at the University of Michigan was conducted. Patients who filled out a pre- and at least one postoperative EPIC survey and had surgery by a urologic oncologist who had performed at least 150 robotic RP were included in the analysis.

\section{Exposure and outcome measurement}

The primary exposure variable was whether an AU stitch was performed, which was recorded in the database by the surgeon immediately after the procedure. The AU stitch is performed after occlusion of the dorsal venous complex. A 2-0 braided absorbable suture is placed through the anterior urethra and tied; the needle of the suspension suture is then brought through the periosteum of the posterior pubis and secured with an absorbable clip. This stitch is illustrated in Figure 1, where robotic arm 1 is retracting the anterior prostate and arm 2 is caudad to the AU stitch. The absorbable suture clip is seen securing the suture to the periosteum.

The first 142 patients included in the study had surgery before September 27, 2007, and did not undergo AU. The next 87 had an AU stitch performed. The patient was blinded to the presence or absence of an AU stitch. Secondary exposures were abstracted from the database and included age, body mass index, estimated blood loss, operative time, prostate weight, age, surgical margin status, Gleason score, complications, and nerve sparing.

Pre- and postoperative EPIC surveys were used to assess urinary and sexual outcomes after prostatectomy by AU status. EPIC is a validated HRQOL instrument based on the UCLA Prostate Cancer Index. ${ }^{11}$ EPIC is designed to evaluate patients receiving treatment for localized prostate cancer, and assesses four domains of functioning: urinary (U), sexual (S), bowel (B), and hormonal $(\mathrm{H})$. The urinary domain is further subdivided to allow for discrimination between incontinence (UIN) and irritative (UIR) symptoms. Scores for each domain are standardized and range from 0 to 100 with higher scores corresponding to better perceived functional outcomes and satisfaction in patients.

EPIC surveys were administered to patients at baseline before surgery, as well as at $\sim 3$-month intervals after pros-

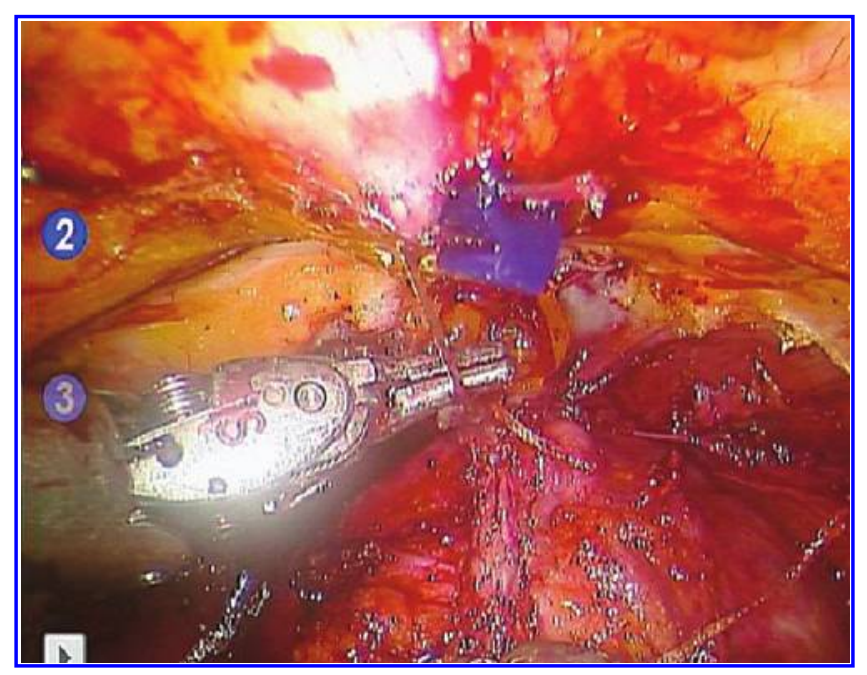

FIG. 1. Anterior urethropexy (AU) stitch. tatectomy. The primary outcome of interest was urinary function as measured by the EPIC-UIN and EPIC-UIR subdomains; an important secondary outcome was sexual function, measured by the EPIC-S domain.

\section{Statistical analysis}

Patients were stratified by AU status. Continuous variables were compared using the Student's $t$-test or Wilcoxon rank sum test. Categorical variables were compared using the general or Mantel-Haenszel chi-square test. A paired $t$-test was used to compare mean UIN, UIR score, and sexual domain scores between the AU and no AU groups at 3-month intervals.

A generalized estimating equations linear mixed model was then used to compare the rate of change of EPIC UIN, UIR, and sexual domain score after robotic RP. The model controlled for age, baseline EPIC scores, Gleason Score, nerve sparing status, complication status, margin status, body mass index, prostate weight, and pathologic stage in all patients. All reported comparisons are made using this adjusted model. The subdomains of UIN and UIR were assessed separately, as was EPIC-S.

Significance was set at $p<0.05$ using standard statistical software (SAS, ver 9.1.2; Cary, NC). This research was approved by the Institutional Review Board at the University of Michigan.

\section{Results}

\section{Baseline patient characteristics}

Of the 229 patients in the study, 87 did have and 142 did not have an AU performed. Of those who completed preand postoperative surveys, complete data were available for 224 patients from 413 surveys for UIN, 215 patients from 392 surveys for UIR, and 219 patients from 412 surveys for EPIC-S.

Baseline characteristics for the patient population are described in Table 1. Overall, patients who had robotic RP without AU were more likely to have Gleason 8 disease and T3 disease and less likely to have bilateral nerve sparing (BNS) during the procedure. Patients undergoing AU also tended to have lower blood loss and were somewhat younger than the non-AU group. There were no baseline differences in mean operative time, prostate weight, complication status, or margin status (including apical margin).

Additionally, no baseline differences were observed in urinary continence or sexual function when comparing patients who had complete data for each domain. A mild baseline statistical difference did exist for irritative symptoms, although the magnitude of the difference was of questionable clinical significance (EPIC-UIR $=90.0$ vs. 83.9, $p=0.003$; Table 2).

\section{Urinary outcomes}

Postoperatively, both groups showed an expected decrease in UIN at 3 months compared with baseline, with a subsequent rise thereafter, which is depicted in Figure 2(a). After adjustment, the AU group, however, demonstrated a higher average score at 3 months $(E P I C-U I N=68)$ than the non-AU group (EPIC-UIN $=58$ ), reflecting better urinary continence function at this early time point $(p=0.015)$. UIN scores at all 
Table 1. Patient Characteristics

\begin{tabular}{|c|c|c|c|}
\hline & $A U(\mathrm{n}=87)$ & No-AU $(\mathrm{n}=142)$ & $\mathrm{p}$-Value \\
\hline \multicolumn{4}{|l|}{ BMI } \\
\hline Mean (SE) & $29.43(0.86)$ & $30.25(0.57)$ & \multirow[t]{2}{*}{0.06} \\
\hline Median & 28.07 & 28.94 & \\
\hline \multicolumn{4}{|c|}{ Blood loss (mL) } \\
\hline Mean (SE) & 139.48 (11.60) & 207.33 (15.65) & \multirow[t]{2}{*}{0.008} \\
\hline Median & 100.00 & 150.00 & \\
\hline \multicolumn{4}{|c|}{ OR time (hour) } \\
\hline Mean (SE) & $3.65(0.05)$ & $3.77(0.06)$ & \multirow[t]{2}{*}{0.28} \\
\hline Median & 3.58 & 3.68 & \\
\hline \multicolumn{4}{|c|}{ Prostate weight $(\mathrm{g})$} \\
\hline Mean (SE) & $45.44(1.52)$ & $48.62(1.41)$ & \multirow[t]{2}{*}{0.24} \\
\hline Median & 42.46 & 44.18 & \\
\hline \multicolumn{4}{|l|}{ Age } \\
\hline Mean (SE) & $58.53(0.86)$ & $61.08(0.58)$ & \multirow[t]{2}{*}{0.01} \\
\hline Median & 58.57 & 61.55 & \\
\hline \multicolumn{4}{|c|}{ Follow-up time (months) } \\
\hline Mean (SE) & $8.78(0.44)$ & $8.27(0.27)$ & \multirow[t]{2}{*}{0.01} \\
\hline Median & 11.63 & 9.15 & \\
\hline \multicolumn{4}{|c|}{ Gleason score } \\
\hline$<6$ & $32(36.78 \%)$ & $31(21.83 \%)$ & \multirow[t]{3}{*}{0.004} \\
\hline 7 & $54(62.07 \%)$ & $102(71.83 \%)$ & \\
\hline$>8$ & $1(1.15 \%)$ & $9(6.34 \%)$ & \\
\hline \multicolumn{4}{|c|}{ Nerve sparing status } \\
\hline Bilateral & $62(71.26 \%)$ & $76(53.52 \%)$ & \multirow[t]{3}{*}{0.02} \\
\hline Unilateral & $11(12.64 \%)$ & $23(16.20 \%)$ & \\
\hline None & $14(16.09 \%)$ & $43(30.28 \%)$ & \\
\hline \multicolumn{4}{|l|}{ Complication } \\
\hline Yes & $16(18.39 \%)$ & $30(21.13 \%)$ & \multirow[t]{2}{*}{0.62} \\
\hline No & $71(81.61 \%)$ & $112(78.87 \%)$ & \\
\hline \multicolumn{4}{|l|}{ Path stage } \\
\hline $\mathrm{T} 2$ & $82(94.25 \%)$ & $115(80.99 \%)$ & \multirow[t]{3}{*}{0.005} \\
\hline T3 & $5(5.75 \%)$ & $26(18.31 \%)$ & \\
\hline $\mathrm{T} 4$ & $0(0 \%)$ & $1(0.70 \%)$ & \\
\hline \multicolumn{4}{|c|}{ Surgical margin positive } \\
\hline Yes & $12(13.79 \%)$ & $28(19.72 \%)$ & \multirow[t]{2}{*}{0.25} \\
\hline No & $75(86.21 \%)$ & $114(80.28 \%)$ & \\
\hline \multicolumn{4}{|c|}{ Apical margin positive } \\
\hline Yes & $4(4.60 \%)$ & $14(9.86 \%)$ & \multirow[t]{2}{*}{0.15} \\
\hline No & $83(95.40 \%)$ & $128(90.14 \%)$ & \\
\hline
\end{tabular}

$\mathrm{AU}=$ anterior urethropexy; $\mathrm{BMI}=$ body mass index; $\mathrm{SE}=$ standard error; $\mathrm{OR}=$ operating room.

other time points and overall were no different when comparing AU versus no AU groups. Larger prostate size $(p=0.003)$ and lower baseline UIN $(p=0.05)$ were associated with lower mean UIN over the 12-month postoperative time period examined.

Although there was a difference in urgency scores (UIR) preoperatively, this difference disappeared after prostatectomy (Fig. 2b). Comparison of all other time points and overall scores revealed no other statistically significant differences in UIR based on surgical approach. Higher baseline UIR score was associated with higher mean postoperative UIR ( $p \leq 0.0001)$ regardless of surgical approach.

\section{Sexual outcomes}

Sexual domain scores over time are illustrated in Figure 2c. EPIC sexual scores were significantly better in the AU group at 3 months $(p=0.002)$ and 6 months $(p=0.006)$. This difference reversed between the groups briefly at 9 months, but
Table 2. Baseline Expanded Prostate Cancer Index COMPOSITE SCORES

\begin{tabular}{llllc}
\hline & \multicolumn{1}{c}{$A U$} & \multicolumn{1}{c}{ No- $A U$} & p-value & Total \\
\hline Baseline & $95.3(1.13)$ & $93.7(1.04)$ & 0.32 & $94.3(0.78)$ \\
$\quad$ UIN (SE) & & & & \\
$n=224$ & $n=85$ & $n=139$ & & \\
Baseline & $90.0(1.39)$ & $83.9(1.45)$ & 0.003 & $86.3(1.05)$ \\
$\quad$ UIR (SE) & & & & \\
$n=215$ & $n=84$ & $n=131$ & & \\
Baseline sex & $76.9(2.71)$ & $71.5(2.46)$ & 0.16 & $73.6(1.84)$ \\
$\quad$ score (SE) & & & & \\
$n=219$ & $n=84$ & $n=135$ & & \\
\hline
\end{tabular}

$\mathrm{UIN}=$ incontinence; UIR $=$ irritative.

did not achieve statistical significance at this time point, and sexual scores were again improved in the AU group compared with the no-AU group at 12 months $(p=0.004)$. Predictors of higher sexual domain scores after robotic RP included having an AU performed, presence of nerve sparing, and having higher baseline sexual domain score.

\section{Discussion}

Patients who received an AU stitch during robotic prostatectomy demonstrated an improvement in continence at 3 months after surgery. Although this urinary difference disappeared by 6 months of follow-up, AU patients were noted to have better sexual function earlier in their recovery process. The AU stitch did not show an effect on postoperative irritative urinary symptoms.

The finding that an AU stitch improves early patient-reported urinary continence is encouraging, and reflects the anatomic theory that the use of this stitch increases urethral support. The 10-point average difference in EPIC scores that we found at the 3-month follow-up time was not just statistically significant, but also represents a differential that is thought to be clinically significant. ${ }^{5}$ The explanation for this finding is that the AU suspension stitch provides early anatomic urethral support, which is later provided by tissue healing. Earlier return of continence may allow patients to focus on other aspects of their recovery experience, such as activity and sexual function.

The AU stitch does not appear to have any irritative side effects. The mild difference in EPIC-UIR scores we observed preoperatively disappeared after prostatectomy. This finding is consistent with previous studies showing improvement in urgency scores after prostatectomy. ${ }^{12,13}$ Although the baseline differences in UIR were statistically different, we feel that the differential of 6.1 points is unlikely to be clinically significant. Although urethral support from the AU stitch may cause changes in anatomic angulation, it does not appear to increase bladder reactivity.

The earlier return of sexual function associated with the AU stitch that we observed in the EPIC-S domain is surprising. Patients who underwent AU had improved sexual outcomes compared with the control group at 3,6, and 12 months after surgery. There is no clear anatomic explanation for an AU suspension stitch improving short-term sexual outcomes. We postulate that patients with better urinary continence may be more comfortable initiating sexual activity after surgery, translating into better EPIC-S domain scores. Patients without 


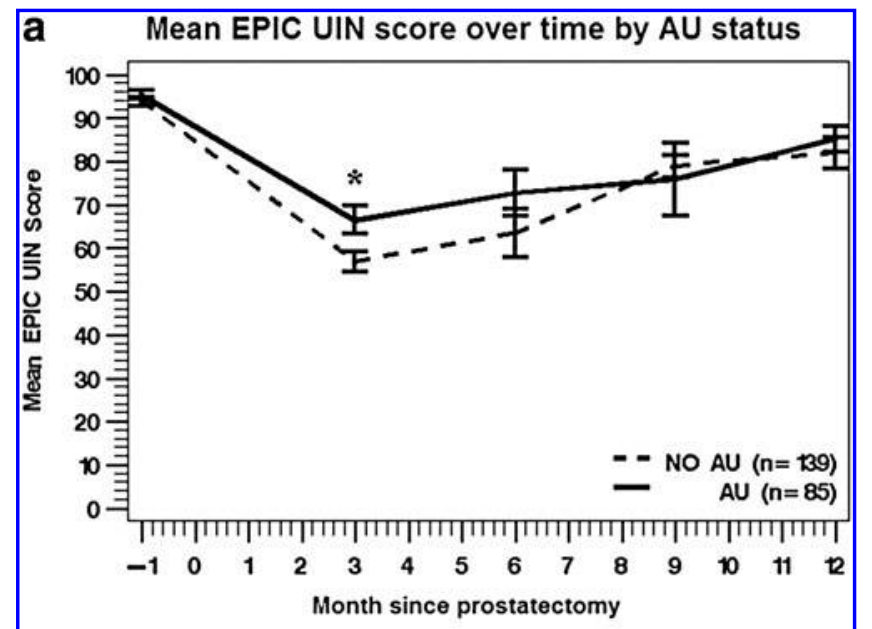

b Mean EPIC UIR score over time by AU status

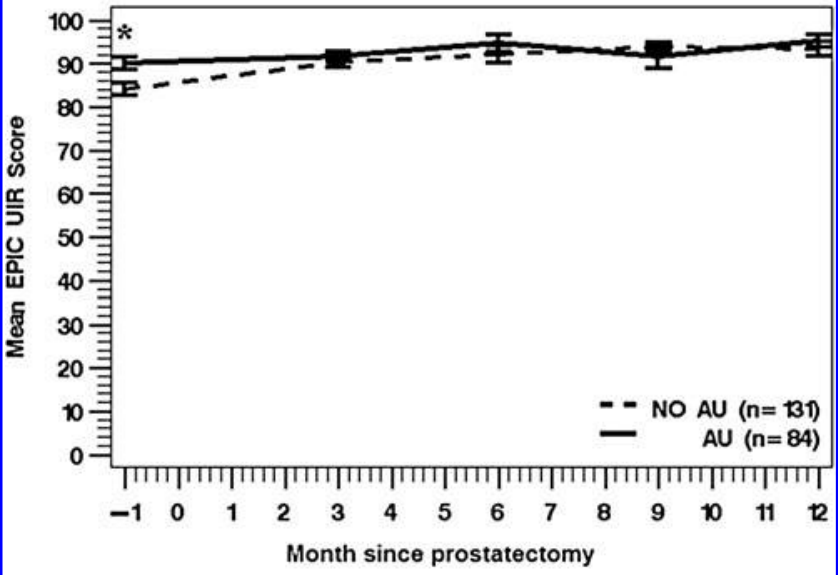

C Mean EPIC sex domain score over time by AU status

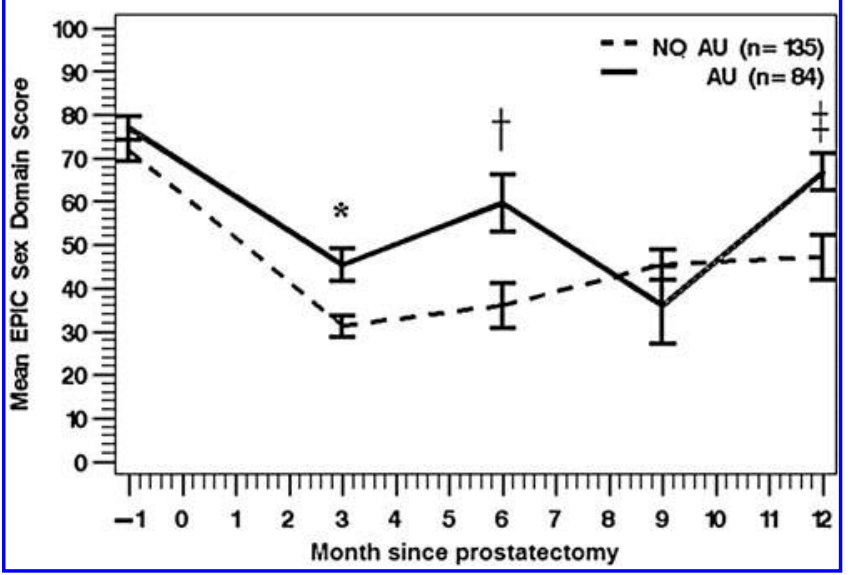

FIG. 2. (a) Change in Expanded Prostate Cancer Index Composite (EPIC) UIN scores over time. ${ }^{*} p=0.015$. (b) Change in EPIC UIR scores over time. ${ }^{*} p=0.011$. (c) Change in EPIC sex scores over time. ${ }^{*} p=0.002 ;{ }^{\dagger} p=0.006$; ${ }^{\star} p=0.004$. $\mathrm{UIN}=$ incontinence; UIR $=$ irritative.

the AU stitch did have scores that surpassed the AU group at the 9 month time-point; however, sexual function scores were again better at 12 months. The brief reversal observed at the 9month follow-up (which is less routine at our institution) may have been because of non-AU patients with early incontinence being followed more closely and whose urinary and sexual function were both improving by this time point. Alternatively, AU patients returning to clinic with specific sexual HRQOL complaints may have also led to a downward bias in scores at this time point.

The finding that a urethral suspension stitch aids return of continence during robotic RP is consistent with previous studies examining a similar stitch during open RP. ${ }^{8,9}$ One group specifically advocates preservation of the puboprostatic ligaments in conjunction with a urethral suspension. ${ }^{14}$ Several studies of robotic prostatectomy have also indicated that anterior urethral support may be important to return of continence. ${ }^{15,16}$ A recent study evaluated the effect of an AU stitch on pad use. ${ }^{10}$ These authors found less pad use in patients having an AU stitch, but did not report sexual function. Our urinary results use the entire EPIC urinary domains that have been validated in evaluating postprostatectomy urinary function. ${ }^{5}$ Our study is also unique in that we describe the effect of AU sexual outcomes after robotic RP.

Our study has several limitations. First, baseline differences existed on univariate analysis in the number of patients who had BNS, with a greater percentage of patients in the AU group receiving $B N S$. However, we did use a multivariate model adjusting for this variable for our comparisons, and the resulting urinary and sexual differences between groups still persisted.

Another consideration for this study is that the consecutive nature of cases might mean that surgeon learning factor was contributing to better outcomes for later cases (i.e., AU cases). Although this could have affected our outcomes, the period over which the data were collected was relatively short, and occurred several years after adopting robotic RP at our institution. Further, the surgeons involved had performed $>150$ robotic cases each by the start of the study period. Our institutional data regarding margin status, continence, and sexual function suggest that the learning curve plateaus at $\sim 100$ cases; the stability of our overall technique during this time makes it unlikely that other technical modifications or learning contributed to the observed differences.

A final limitation is that our dataset lacks specific information regarding the rate of PDE-5 inhibitor use, which could affect our results regarding the rate of sexual recovery after robotic RP. All patients were encouraged to use PDE-5 inhibitors (unless medically contraindicated) postoperatively. This variable could have an unmeasured effect on our sexual recovery results, and merits consideration during future investigations.

The results of this study on robotic prostatectomy patients and other investigators' previously published series ${ }^{8-10,14}$ indicate that the $\mathrm{AU}$ technique merits consideration during robotic prostatectomy. Additional investigation to strengthen the causality of the AU stitch to the observed outcomes could include a prospective evaluation. This would better control patient selection and variation in nerve sparing. As our robotic prostatectomy experience continues to evolve, we will examine if the AU stitch offers a long-term benefit to our patients.

\section{Conclusions}

At our institution, AU during robotic RP leads to improved urinary continence and sexual functioning at 3 months of follow-up. This earlier return to continence may facilitate an earlier return to sexual activity also observed in this group. 
The AU stitch may therefore offer a short-term quality-of-life advantage for patients undergoing robotic RP.

\section{Disclosure Statement}

No competing financial interests exist.

\section{References}

1. Bianco FJ Jr., Scardino PT, Eastham JA. Radical prostatectomy: Long-term cancer control and recovery of sexual and urinary function ("trifecta"). Urology 2005;66(5 Suppl): 83-94.

2. Brown JA, Elliott DS, Barrett DM. Postprostatectomy urinary incontinence: A comparison of the cost of conservative versus surgical management. Urology 1998;51:715-720.

3. Lev EL, Eller LS, Gejerman G, et al. Quality of life of men treated for localized prostate cancer: Outcomes at 6 and 12 months. Support Care Cancer 2009;17:509-517.

4. Fulmer BR, Bissonette EA, Petroni GR, Theodorescu D. Prospective assessment of voiding and sexual function after treatment for localized prostate carcinoma: Comparison of radical prostatectomy to hormonobrachytherapy with and without external beam radiotherapy. Cancer 2001;91:2046-2055.

5. Sanda MG, Dunn RL, Michalski J, et al. Quality of life and satisfaction with outcome among prostate-cancer survivors. N Engl J Med 2008;358:1250-1261.

6. Walsh PC, Mostwin JL. Radical prostatectomy and cystoprostatectomy with preservation of potency. Results using a new nerve-sparing technique. Br J Urol 1984;56:694-697.

7. Razi A, Yahyazadeh SR, Sedighi Gilani MA, Kazemeyni SM. Bladder neck preservation during radical retropubic prostatectomy and postoperative urinary continence. Urol J 2009; 6:23-26.

8. Campenni MA, Harmon JD, Ginsberg PC, Harkaway RC. Improved continence after radical retropubic prostatectomy using two pubo-urethral suspension stitches. Urol Int 2002; 68:109-112.

9. Noguchi M, Shimada A, Nakashima O, Kojiro M, Matsuoka $\mathrm{K}$. Urodynamic evaluation of a suspension technique for rapid recovery of continence after radical retropubic prostatectomy. Int J Urol 2006;13:373-378.

10. Patel VR, Coelho RF, Palmer KJ, Rocco B. Periurethral suspension stitch during robot-assisted laparoscopic radical prostatectomy: Description of the technique and continence outcomes. Eur Urol 2009;56:472-478.
11. Wei JT, Dunn RL, Litwin MS, Sandler HM, Sanda MG. Development and validation of the expanded prostate cancer index composite (EPIC) for comprehensive assessment of health-related quality of life in men with prostate cancer. Urology 2000;56:899-905.

12. Kumar V, Toussi H, Marr C, Hough C, Javle P. The benefits of radical prostatectomy beyond cancer control in symptomatic men with prostate cancer. BJU Int 2004;93: 507-509.

13. Lepor H, Kaci L. The impact of open radical retropubic prostatectomy on continence and lower urinary tract symptoms: A prospective assessment using validated selfadministered outcome instruments. J Urol 2004;171:12161219.

14. Avant OL, Jones JA, Beck H, Hunt C, Straub M. New method to improve treatment outcomes for radical prostatectomy. Urology 2000;56:658-662.

15. Tewari A, Jhaveri J, Rao S, et al. Total reconstruction of the vesico-urethral junction. BJU Int 2008;101:871-877.

16. Tewari AK, Bigelow K, Rao S, et al. Anatomic restoration technique of continence mechanism and preservation of puboprostatic collar: A novel modification to achieve early urinary continence in men undergoing robotic prostatectomy. Urology 2007;69:726-731.

Address correspondence to: Emilie K. Johnson, M.D. Department of Urology University of Michigan 3875 Taubman Center

1500 East Medical Center Drive

Ann Arbor, MI 48109-5330

E-mail: emiliej@med.umich.edu

$\begin{aligned} & \quad \text { Abbreviations Used } \\ & \mathrm{AU}=\text { anterior urethropexy } \\ & \mathrm{BMI}=\text { body mass index } \\ & \mathrm{BNS}=\text { bilateral nerve sparing } \\ & \mathrm{EPIC}=\text { The Expanded Prostate Cancer Index Composite } \\ & \mathrm{HRQOL}=\text { health-related quality of life } \\ & \mathrm{OR}=\text { operating room } \\ & \mathrm{RP}=\text { radical prostatectomy } \\ & \mathrm{SE}=\text { standard error }\end{aligned}$


